\title{
Picture the future: emerging imaging modalities
}

\author{
Authors: Jean SZ Lee ${ }^{A}$ and Fergus V Gleeson ${ }^{B}$
}

The past century has witnessed accelerated development in imaging modalities. Better anatomical visualisation and improved data analysis have improved survival rates. Through emerging functional, molecular and structural imaging modalities, better anatomical visualisation has been extended to cellular and molecular detail, improving diagnosis and management of diseases. This article reviews the advances made in emerging imaging modalities as well as their potential applications in targeted therapy.

KEYWORDS: Perfusion CT, PET-CT, DWI MRI, HIFU, targeted therapy

\section{Introduction}

Since Wilhelm Röntgen's discovery of X-rays with the first radiograph of his wife's left hand in $1895,{ }^{1}$ the development of imaging modalities has been explosive. From the first clinical computed tomography (CT) scan in $1971,{ }^{2}$ to the threedimensional volume rendering of images from volumetric CT scanners in $1989,{ }^{3}$ to the first integrated positron emission tomography computed tomography (PET-CT) scan in $1998,{ }^{4}$ imaging technologies have allowed better anatomical visualisation of the human body. The demand for imaging examination has also risen exponentially. The number of CT scans performed by NHS trusts has increased from 1.45 million in 2000-2001 to 3.72 million in 2009-2010. In 2010, NHS England owned a total of 426 CT scanners, 376 of which were installed in the last 10 years. ${ }^{5}$ Today, emerging imaging modalities that incorporate improved data analysis, functional, molecular and structural imaging, and that facilitate imagetargeted therapy, are set to further transform the practice of medicine.

\section{Improved data analysis}

The use of imaging technology with improved data analysis can reduce the projected rise of cancer deaths, which is predicted to

Authors: ${ }^{\text {A }}$ specialty registrar (academic clinical fellow) in clinical radiology, Radiology Department, Oxford University Hospitals NHS Trust, Oxford, UK; ${ }^{\text {B }}$ radiology consultant, Radiology Department, Oxford University Hospitals NHS Trust, Oxford, UK double from 7.6 million deaths in 2008 to 13.2 million deaths in $2030 .{ }^{6}$ Using a combination of epidemiological data analysis with low dose CT screening, the National Lung Screening Trial has successfully reduced lung cancer mortality. ${ }^{7}$ In total, 53,454 participants at high risk for lung cancer were randomly assigned to receive annual low-dose helical CT scan or chest radiograph screenings for three years and followed up for an additional 3.5 years. Lung cancer mortality was reduced by $20.3 \%$ in the low-dose helical CT group, compared with the chest radiograph group.

Image processing techniques such as maximum-intensityprojection (MIP) have enabled the increased detection of pulmonary nodules on CT scans. ${ }^{8}$ A study comparing the use of MIP with volume rendering (VR) image processing techniques found VR to be superior to MIP in detecting small pulmonary nodules. ${ }^{9}$ These image processing techniques have been further developed using machine learning methods to facilitate computer-aided detection of pulmonary nodules. ${ }^{10,11}$

New diagnostic algorithms have been developed using improved data analysis techniques to improve disease diagnosis and management. By incorporating imaging features, such as size and solidity of the nodules, and clinical and social data such as smoking history, a comprehensive algorithm was developed to stratify patients in the diagnosis and management of solitary pulmonary nodules. ${ }^{12}$ This has led to a more streamlined diagnostic and management pathway, thus reducing unnecessary investigations.

\section{Functional imaging}

Integrated colour perfusion imaging with CT scans has enabled the creation of a quantifiable map of regional tissue perfusion to be displayed according to a colour scale. The rate of enhancement of a region of interest is quantified through a sequence of images rapidly acquired immediately after a bolus of intravenous injection of a contrast medium. First described in 1991, its potential applications have been widespread, as the authors predicted, and has proven to be useful in studies of intrarenal blood flow, regional perfusion in tumours and studies of cerebral perfusion in patients with stroke. ${ }^{13}$ This has further informed clinical decision making by enabling the prediction of tumour behaviour and stroke recovery, as well as by stratifying benign or malignant renal tumours. For example, a study of the use of perfusion CT in rectal cancer has shown that tumours with short mean transit time (MTT) values tended to respond poorly to chemotherapy and radiation therapy. ${ }^{14}$ 
Fig 1. Xe-MRI ventilation images and lung density images showing abnormal heterogenous signal throughout the lungs. Abstracted XeMRI ventilation maps ( $b$ and $d$ ) are mapped onto conventional MRI (a) and volumetric CT data (c) to produce 3D ventilation images (e and $\mathrm{f}$ ). Xe-MRI = xenon magnetic resonance imaging.
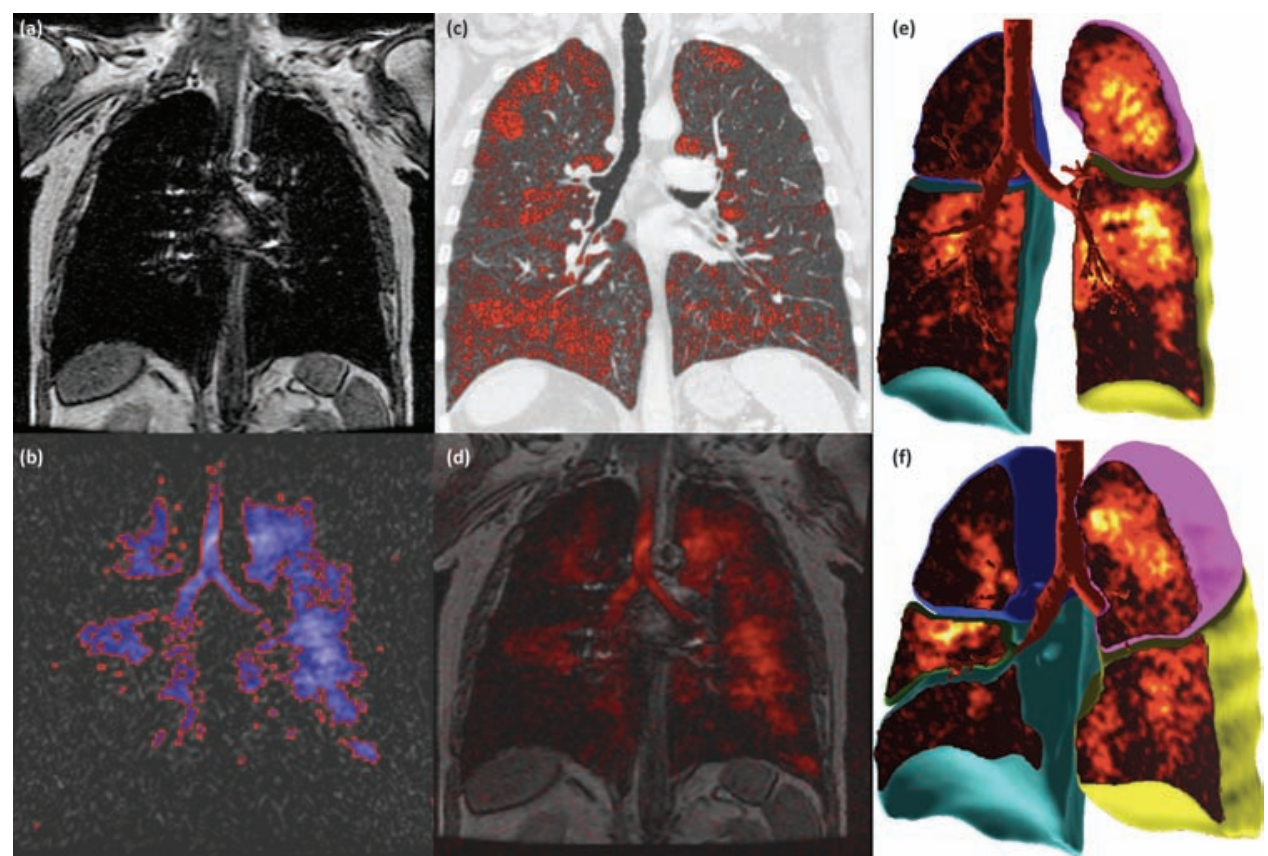

Functional imaging of tumours using dynamic contrastenhanced magnetic resonance imaging (DCE-MRI) has also been used to predict tumour response to treatment. A more comprehensive visualisation of the wash-in and wash-out contrast kinetics within tumours has allowed pharmacokinetic modeling to generate a DCE-MRI biomarker, $\Delta \mathrm{K}^{\text {trans }}$. DCEMRI has been used in early phase trials to determine a minimal effective dose of chemotherapy. ${ }^{15,16}$ In a study of women with breast cancer, $\Delta \mathrm{K}^{\text {trans }}$ was shown to be able to discriminate non-responders, partial responders, and good responders to bevacizumab. These findings were corroborated by the authors' pathological findings. ${ }^{17}$ DCE-MRI has also been shown to be accurate for detecting residual local disease following chemoradiotherapy in patients with locally advanced breast cancer. ${ }^{18}$

MRI using hyperpolarised noble gas is emerging as a new technique to describe regional functional abnormality in the lung. The MR signal originates from hyperpolarised noble gas, which is inhaled into the lungs. The highly diffusible helium gas $\left({ }^{3} \mathrm{He}\right)$ has been investigated in normal patients as well as in patients with lung disease such as asthma, cystic fibrosis and chronic obstructive pulmonary disease. It has also been used in patients after radiation therapy. The poor availability of ${ }^{3} \mathrm{He}$ and its cost have led researchers to investigate an alternative noble gas, xenon $\left({ }^{129} \mathrm{Xe}\right)$. This is a highly soluble gas which has been used in pulmonary and cerebral imaging. ${ }^{19}$ Regional ventilation and alveolar size can be quantified using the distribution and diffusion of the gas, respectively. As a small fracture of the inhaled, ${ }^{129} \mathrm{Xe}$ is taken up by the lung parenchyma and blood. Xenon MR can distinguish the resulting signals and may lead to a new way of performing ventilation/perfusion scans by providing a real-time picture of lung function (Fig 1). ${ }^{20}$

\section{Molecular imaging}

The advent of PET-CT scans has enabled more detailed imaging of the body at a molecular and cellular level. PET-CT scans with a radioactive tracer 18 -flurodeoxyglucose $\left({ }^{18} \mathrm{FDG}\right)$ allow the quantification of metabolically active cells in a colour-encoded map. The use of PET-CT scans in pre-operative assessment of patients with suspected non-small-cell lung cancer has improved the differentiation of benign and malignant pulmonary nodules and the detection of distant metastasis, leading to a $50 \%$ reduction in futile thoracotomies in patients. ${ }^{21}$ The use of PET-CT scans in a randomised controlled trial also improved the overall survival of patients with early lung cancer (hazard ratio, 0.88; confidence interval, 0.61-1.29). ${ }^{22}$

New PET-CT tracers are also under development. Cholinebased ${ }^{18} \mathrm{~F}$-labelled tracers have been shown to be useful in distinguishing malignant and normal cerebral tissue and in detecting local recurrence of prostate cancer. ${ }^{23}$ PET-CT ${ }^{18} \mathrm{~F}$-Fluoride scan is also the most sensitive and specific for detecting bone metastases in patients with prostate cancer. ${ }^{24}$ Liver imaging with a novel non-invasive MR method allows the rapid characterisation of liver tissue, including the quantification of hepatic iron and fat content, as well as the identification and staging of inflammation and fibrosis. The high corroboration with pathological findings suggests that this method could potentially replace liver biopsy for many indications. $^{25}$

The first trial of metabolic imaging using dynamic nuclear polarisation of $\left[1-{ }^{13} \mathrm{C}\right]$ pyruvate in humans has shown that it is safe and useful in non-invasively characterising changes in tumour metabolism in patients with prostate cancer. Regions of biopsy-proven cancer were found to have elevated levels of the hyperpolarised tracer. ${ }^{26}$

\section{Combined functional and molecular imaging}

Trials investigating the combined use of functional and molecular imaging to better visualise regions of active angiogenesis are underway. PET-CT scans with ${ }^{18} \mathrm{~F}$-FDG uptake and tumour perfusion parameters from perfusion CT scans 

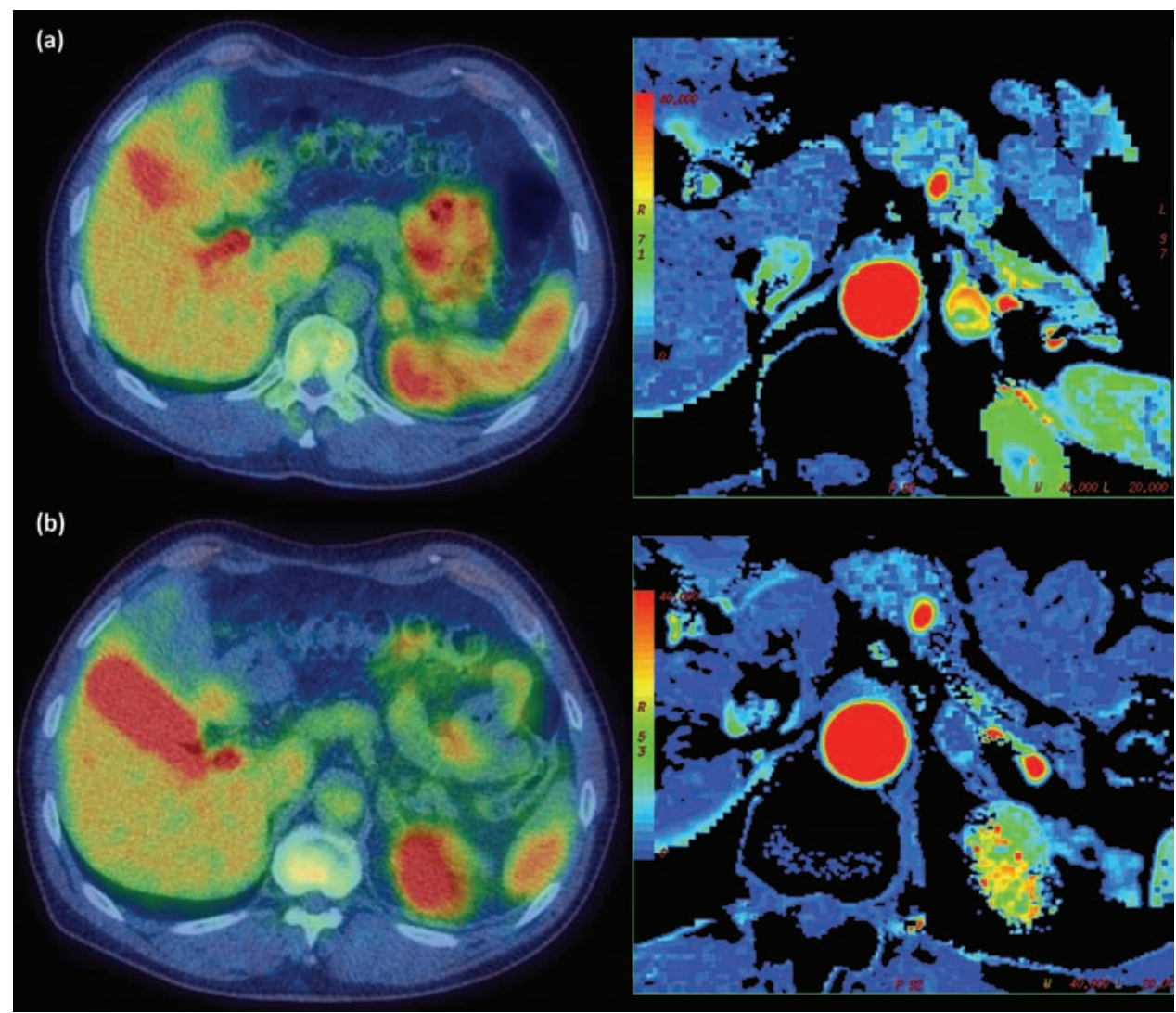

Fig 2. Axial slices showing PET-CT (left) and perfusion CT (right) images of an adrenal metastasis before (above) and after (after) treatment with an anti-angiogenic agent. PET-CT = positron emission tomographycomputed tomography.

such as blood flow, blood volume and $\mathrm{K}^{\text {trans }}$ have both been shown to correlate with tumour biomarkers such as Ki67 and microvessel density, respectively. ${ }^{27}$ As such, the combined use of functional and molecular imaging can provide complementary data to improve tumour profiling.

A new PET tracer, fluciclatide, is a small peptide containing the Arg-Gly-Asp (RGD) tripeptide that has been shown to have high affinity for integrin $\alpha_{v} \beta_{3}$ receptors, which are upregulated in metastatic tumour cells and in angiogenesis. It is being investigated in combination with perfusion CT scans to quantify tumour response to anti-angiogenic agent sunitinib in patients with metastatic renal cell cancer in Oxford (NCT01492192; Fig 2).

\section{Structural imaging}

MRI diffusion-weighted imaging allows the visualisation and quantification of the diffusivity of water molecules within tissue, which may be altered by restricting barriers owing to pathologic processes. This imaging technology therefore allows the histopathological appearances of the tissue to be visualised non-invasively. A study comparing the appearances of colorectal hepatic metastases on diffusion-weighted MRI with histopathology revealed that colorectal liver metastases with high signal rim intensity on imaging correlate with central necrosis on tissue sections. ${ }^{28}$

A trial comparing PET, conventional contrast-enhanced CT, perfusion CT and diffusion-weighted imaging (DWI) MRI with outcome, histological markers of angiogenesis and genomic analysis in colorectal hepatic metastasis, is due to recruit in Oxford (Fig 3).

Image post-processing of ultrasound images has also enabled better structural imaging. For example, the intensity of the reflected B-mode ultrasound signal is correlated with lipid content of carotid artery plaques, which may be associated with intraplaque haemorrhage. ${ }^{29}$ Nakagami parametric imaging, which is based on the distribution of back-scattered radiofrequency echoes, has been used to measure the degree of change in tissue caused by ultrasound-induced thermal exposures. ${ }^{30}$ In addition, the use of power Doppler and contrast-enhanced ultrasound can allow the visualisation of the vascularity and perfusion characteristics of a lesion in vivo, respectively. Acoustic radiation force impulse (ARFI) elastography has been shown to be a reliable method in assessing tissue stiffness and therefore liver fibrosis. ${ }^{31}$

\section{Therapies using modern imaging technologies}

In addition to image-guided ablative techniques, such as radiofrequency or microwave ablation, high intensity focused ultrasound (HIFU) is a promising new local image-guided treatment. HIFU can generate mild hyperthermia at intensities lower than those required for ablation, resulting in less adverse events from hyperthermia compared with the current ablative treatments. Celsion and the University of Oxford will shortly begin recruiting for the first human trial of the targeted 
Fig 3. Axial images of (clockwise from top left) PET-CT, perfusion CT, DWI MRI and conventional contrastenhanced CT images of a patient with a hepatic metastasis from colorectal cancer. DWI MRI = diffusion-weighted imaging magnetic resonance imaging; PET-CT = positron emission tomography-computed tomography.

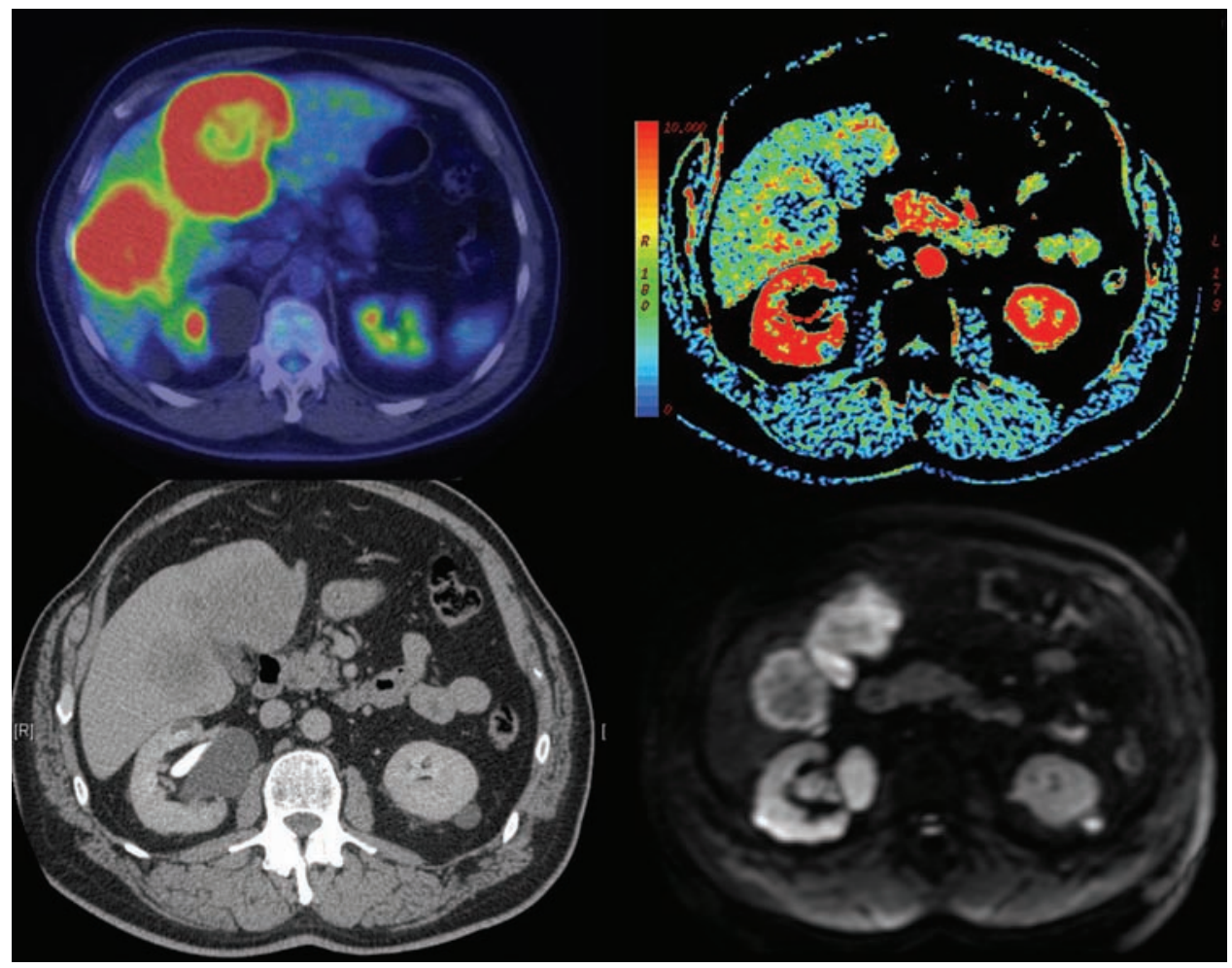

delivery of chemotherapy. In this study, doxorubicin will be delivered intravenously, covered in a liposomal heat sensitive shell that will allow drug release when heated using HIFU. This would enable targeted delivery of the chemotherapy within the lesion of interest as well as reduced systemic administration of chemotherapy and its adverse effects. ${ }^{32}$

\section{Conclusion}

The exponential development and demand for imaging examinations has led to the development of emerging imaging modalities. These imaging modalities have the potential to add to our structural, molecular and functional understanding of pathological processes in addition to providing novel ways to deliver targeted therapy. The results of ongoing and future trials in these emerging modalities will transform our current clinical pathways and are keenly awaited.

\section{References}

1 Röntgen WC. December 28, 1895: On A New Kind of Rays. Vet Radiol Ultrasound 1995;36:371-4.

2 Hounsfield GN. Computerized transverse axial scanning tomography: Part-1, description of the system. Br J Radiol 1973;46: 1016-22.

3 Kalender WA, Seissler W, Vock P. Single-breath-hold spiral volumetric CT by continuous patient translation and scanner rotation. Radiology 1989;173:414.

4 Beyer T, Townsend D, Brun T et al. A combined PET/CT scanner for clinical oncology. J Nucl Med 2000;41:1369-79.

5 National Audit Office. Department of Health: Managing high value capital equipment in the NHS in England. London: NAO, 2011.
6 Jemal A, Bray F, Ferlay J, Ward E, Forman D. Global cancer statistics. CA Cancer J Clin 2011;61:69-90.

7 The National Lung Screening Trial Research Team. Reduced lungcancer mortality with low-dose computed tomographic screening. N Engl J Med 2011;365:395-409.

8 Gruden JF, Norris SD, Klausner TS. Incremental benefit of maximum- intensity-projection images on observer detection of small pulmonary nodules revealed by multidetector CT. Am J Roentgenol 2002;179:149-57.

9 Peloschek P, Sailer J, Weber M, Herold CJ, Prokop M, Schaeferprokop C. Pulmonary nodules: sensitivity of maximum intensity projection versus that of volume rendering of $3 \mathrm{D}$ multidetector CT data. Radiology 2007;243:561-9.

10 Rubin GD, Lyo JK, Paik DS et al. Pulmonary nodules on multidetector row CT scans : performance comparison. Radiology 2005;234:274-83.

11 Zhao Y, de Bock GH, Vliegenthart R et al. Performance of computer-aided detection of pulmonary nodules in low-dose CT: comparison with double reading by nodule volume. Eur Radiol 2012;22:2076-84.

12 Patel VK, Naik SK, Naidich DP et al. A practical algorithmic approach to the diagnosis and management of solitary pulmonary nodules: part 2: pretest probability and algorithm. Chest 2013;143:840-6.

13 Miles K, Hayball M, Dixon A. Colour perfusion imaging: a new application of computed tomography. Lancet 1991;337:643-5.

14 Sahani, D VKalva SP, Hamberg LM et al. Radiology and treatment response in rectal cancer with multisection CT : Initial. Proc Natl Acad Sci USA 2005;234:785-92.

15 Mross K, Drevs J, Müller M et al. Phase I clinical and pharmacokinetic study of PTK/ZK, a multiple VEGF receptor inhibitor, in patients with liver metastases from solid tumours. Eur J Cancer 2005;41:1291-9. 
16 Liu G, Rugo HS, Wilding G et al. Dynamic contrast-enhanced magnetic resonance imaging as a pharmacodynamic measure of response after acute dosing of AG-013736, an oral angiogenesis inhibitor, in patients with advanced solid tumors: results from a phase I study. J Clin Oncol 2005;23:5464-73.

17 De Bazelaire C, Calmon R, Thomassin I et al. Accuracy of perfusion MRI with high spatial but low temporal resolution to assess invasive breast cancer response to neoadjuvant chemotherapy: a retrospective study. BMC Cancer 2011;11:361.

18 Drew PJ, Kerin MJ, Mahapatra T et al. Evaluation of response to neoadjuvant chemoradiotherapy for locally advanced breast cancer with dynamic contrast-enhanced MRI of the breast. Eur J Surg Oncol 2001;23:617-20.

19 Kauczor H, Surkau R, Roberts T. MRI using hyperpolarized noble gases. Eur Radiol 1998;8:820-7.

20 Mugler JP, Altes TA, Ruset IC et al. Simultaneous magnetic resonance imaging of ventilation distribution and gas uptake in the human lung using hyperpolarized xenon-129. Proc Natl Acad Sci USA 2010;107:21707-12.

21 Van Tinteren H, Hoekstra OS, Smit EF et al. Effectiveness of positron emission tomography in the preoperative assessment of patients with suspected non-small-cell lung cancer: the PLUS multicentre randomised trial. Lancet 2002;359:1388-93.

22 Maziak DE, Darling GE, Inculet RI et al. Positron emission tomography in staging early lung cancera randomized trial. Ann Intern Med 2009;151:221-8.

23 Mertens K, Slaets D, Lambert B, Acou M, De Vos F, Goethals I. PET with (18)F-labelled choline-based tracers for tumour imaging: a review of the literature. Eur J Nucl Med Mol Imaging 2010;37:2188-93.

24 Even-Sapir E, Metser U, Mishani E, Lievshitz G, Lerman H, Leibovitch I. The detection of bone metastases in patients with high-risk prostate cancer : 99m Tc-MDP Planar. J Nucl Med 2006;47:287-97.
25 Banerjee R, Pavlides M, Tunnicliffe EM et al. Multiparametric magnetic resonance for the non-invasive diagnosis of liver disease. $J$ Hepatol 2014;60:69-77.

26 Nelson SJ, Kurhanewicz J, Vigneron DB et al. Metabolic imaging of patients with prostate cancer using hyperpolarized [1-13C]pyruvate. Sci Transl Med 2013;5:198ra108.

27 Sauter AW, Winterstein S, Spira D et al. Multifunctional profiling of non-small cell lung cancer using 18F-FDG PET/CT and volume perfusion CT. J Nucl Med 2012;53:521-9.

28 Scurr ED, Collins DJ, Temple L, Karanjia N, Leach MO, Koh D-M. Appearances of colorectal hepatic metastases at diffusion-weighted MRI compared with histopathology: initial observations. $\mathrm{Br} \mathrm{J}$ Radiol 2012;85:225-30.

29 Grønholdt ML, Wiebe BM, Laursen H, Nielsen TG, Schroeder TV, Sillesen H. Lipid-rich carotid artery plaques appear echolucent on ultrasound B-mode images and may be associated with intraplaque haemorrhage. Eur J Vasc Endovasc Surg 1997;14:439-45.

30 Rangraz P, Behnam H, Tavakkoli J. Nakagami imaging for detecting thermal lesions induced by high-intensity focused ultrasound in tissue. Proc Inst Mech Eng Part H J Eng Med 2014;228:19-26.

31 Bota S, Herkner H, Sporea I et al. Meta-analysis: ARFI elastography versus transient elastography for the evaluation of liver fibrosis. Liver Int 2013;33:1138-47.

32 Oxford Biomedical Research Centre. Surgical Innovation and Evaluation - TARDOX Project. Available online from: http:// oxfordbrc.nihr.ac.uk/surgical/sub-themes/tardox-project/ [Accessed 21 April 2014].

Address for correspondence: Prof FV Gleeson, Oxford University Hospitals NHS Trust, Radiology Department, Churchill Hospital, Old Road, Oxford OX3 7LE, UK. Email: fgleeson@mac.com 